\title{
Surgeon-performed Ultrasound in Preoperative Parathyroid Localization: The Cutting Edge of Endocrine Surgeon
}

\author{
${ }^{1}$ Yahya MA, ${ }^{2}$ Normayah K, ${ }^{3}$ Hisham AN \\ ${ }^{1}$ Surgeon, Department of Breast and Endocrine Surgery, Hospital Putrajaya, Precinct 7, 62250 Putrajaya, Malaysia \\ ${ }^{2}$ Surgeon, Department of Breast and Endocrine Surgery, Hospital Putrajaya, Precinct 7, 62250 Putrajaya, Malaysia \\ ${ }^{3}$ Head and Consultant Surgeon, Department of Breast and Endocrine Surgery, Hospital Putrajaya, Precinct 7, 62250 Putrajaya \\ Malaysia
}

Correspondence: Hisham AN, Head and Consultant Surgeon, Department of Breast and Endocrine Surgery, Hospital Putrajaya Precinct 7, 62250 Putrajaya, Malaysia, Phone: +603-88821033, Fax: +603-88831045, e-mail: anhisham@moh.gov.my

\begin{abstract}
Background: Over the years surgery for primary hyperparathyroidism has evolved from bilateral neck exploration to focus unilateral approach. This has been made possible by the advancement in localization technique and availability of the preoperative imaging. This study aimed to determine the feasibility and accuracy of focus unilateral neck approach for primary hyperparathyroidism with surgeonperformed ultrasound as the main decisive preoperative localization imaging technique.

Method: The decision of focus unilateral approach was stipulated if an enlarged parathyroid adenoma was confidently seen in the preoperative localization by surgeon-performed ultrasound. The focus unilateral exploration was terminated if the diseased gland was found. Nonetheless if in doubt, the exploration was extended to include the opposite side of the neck. More importantly any negative preoperative ultrasound will be considered for bilateral neck exploration.

Results: 118 consecutive patients were accrued in this study, 76 females and 42 males with mean age of 50 years. Focus unilateral neck exploration was performed on $86(72.9 \%)$ patients and $96.5 \%$ of them were successfully explored and cured. $13(13.1 \%)$ patients had exploration of both sides of the neck although the initial decision was only to explore one side. The overall cure rate was $93.2 \%$ with the sensitivity and specificity of ultrasound were $78.3 \%$ and $95.1 \%$ respectively.

Conclusion: Surgeon-performed ultrasound in parathyroid localization in coupled with focus unilateral approach in primary HPT can be performed $73 \%$ of patients with a success rate of more than $96 \%$.
\end{abstract}

Keywords: Primary hyperparathyroidism, preoperative localization, surgeon performed ultrasound, unilateral neck exploration.

\section{INTRODUCTION}

Bilateral neck exploration was once considered the gold standard for primary hyperparathyroidism (HPT) with cure rate of more than $95 \%{ }^{1,2}$ In this approach all the four parathyroid glands have to be visualized before any decision is made on which gland or glands to be excised. It is generally agreed that almost $80 \%$ or more of primary HPT is caused by a single adenoma. ${ }^{3}$ Hence in the majority of cases bilateral exploration of both sides of the neck may be possibly unnecessary provided an accurate imaging technique is available. Nowadays, imaging techniques for preoperative localization of parathyroid adenoma has becoming more feasible and readily accessible to surgeons. If the diseased gland can be localized preoperatively, exploration of the predetermined side of the neck is sufficient without the need to explore on both sides.

In this study surgeon-performed ultrasound is used to localize the parathyroid adenoma and hence decide the side of exploration. Ultrasound is used as the localization technique as it is simple, user-friendly and it can be done in the operating room and clinic setting. More importantly it provides a one stop assessment without the need to go through the hustle of getting prior appointment or referral for preoperative ultrasound. We evaluated the feasibility of this approach and its surgical implication on the outcome of our patient diagnosed with primary HPT.

\section{PATIENTS AND METHODS}

All patients diagnosed with primary HPT who had completed and confirmed biochemical diagnosis who had undergone parathyroid surgery between January 2003 and August 2007 were accrued in this study. Ultrasounds were performed using high resolution 5-12 MHz transducer. The examination was performed with the patient in supine position and the neck in extension to optimize visualization of the upper mediastinum. The entire thyroid gland was evaluated first and followed by central compartment of the neck from the level of the submandibular glands to the clavicles on both respective sides. The parathyroid adenoma was identified by its hypoechogenicity, oval shape and encapsulation. The size of the gland was measured by taking its largest two dimensions. 
The precise location of an enlarged parathyroid gland was identified in terms of laterality and superior or inferior position in relation to thyroid gland, with the isthmus as the dividing landmark as well as its relation to upper and lower pole of the thyroid. Focus unilateral neck exploration (UNE) was planned if parathyroid adenoma was visualized during the ultrasound examination and the side of exploration was determined by the ultrasound finding. The diseased gland was recognized intraoperatively by its enlarged size and gross appearance. Operation was terminated if the diseased gland was found and if not, the exploration is extended to include the opposite side of the neck (UNE/BNE). Bilateral neck exploration (BNE) was performed on patients whom the preoperative ultrasound was negative (Fig. 1). Clinical cure is defined when the parathyroid lesion was confirmed to be an adenoma or hyperplasia on paraffin section and the serum calcium level was subsequently normalized and sustained within the normal range for at least 6 months following surgery.

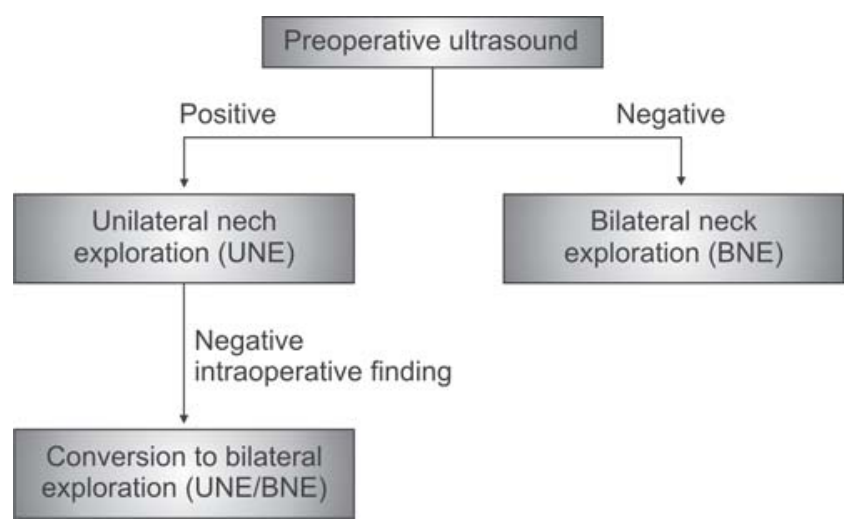

Fig. 1: Options of surgical procedures

\section{RESULTS}

\section{Patients and Pathology}

A total of 123 consecutive patients with primary HPT had undergone parathyroid surgery between January 2003 and August 2007. Out of these, 118 patients who had preoperative ultrasound were included in the study. This involved a total of 467 parathyroid glands, assuming that all patients had four parathyroid glands, with the exceptions of two patients who had previous partial parathyroidectomy. Of the 118 patients, 76 were female and 42 were male and the mean age was 50 , ranging from 16 to 84 years.

The preoperative biochemical investigations are shown in Table 1. Primary HPT was associated with multiple endocrine neoplasia (MEN) in two patients, one patient each for MEN1 and MEN 2A. Ectopic location of parathyroid gland was noted in two patients; one intrathyroidal and the other one in the anterior mediastinum. Examination of the paraffin sections revealed single adenoma in 109 (95.6\%) patients, while the rest were double adenoma in 3 patients and four gland hyperplasia in 2 patients (Fig. 2). The histopathological examinations failed
Table 1: Patient characteristics

\begin{tabular}{lr}
\hline Characteristics & $N=118$ \\
\hline $\begin{array}{l}\text { Age (years), mean } \pm \text { SD } \\
\text { Gender, n (\%) }\end{array}$ & $49.94 \pm 16.78$ \\
- Female & $42(35.4)$ \\
- Male & $3.01(2.41-6.50)$ \\
$\begin{array}{l}\text { Preoperative calcium (mmol/L), } \\
\text { median (range) }\end{array}$ & \\
$\begin{array}{l}\text { Preoperative phosphorus (mmol/L), } \\
\text { median (range) }\end{array}$ & $0.80(0.34-2.16)$ \\
$\begin{array}{l}\text { Preoperative iPTH (pmol/L), } \\
\text { median (range) }\end{array}$ & $35.90(7.6-271.5)$ \\
\hline
\end{tabular}

iPTH = intact parathyroid hormone

\begin{tabular}{|l|c|}
\hline Pathology & N (\%) \\
\hline Single adenoma & $109(95.6)$ \\
\hline Double adenoma & $3(2.6)$ \\
\hline Four gland hyperplasia & $2(1.8)$ \\
\hline
\end{tabular}

Fig. 2: Pathology of primary hyperparathyroidism

to detect parathyroid tissue in specimens of four patients. None of the patients have parathyroid carcinoma.

\section{Surgical Procedures and Outcome}

Ninety-nine patients were planned for unilateral neck exploration. However, 13 patients were decided intraoperatively for exploration on both sides. This gives the conversion rate of $13.1 \%$. The conversions were due to mislead by ultrasound in 12 patients and multiple gland disease in 1 patient. Unilateral exploration was successfully performed in 86 out of total 118 patients (72.9\%). Planned bilateral explorations were performed on 19 patients (16.1\%). 110 patients (93.2\%) were cured from hypercalcemia. In UNE group 83 (96.5\%) patients were cured, while 10 (76.9\%) and 17 (89.5\%) patients in UNE/BNE and BNE groups were cured respectively (Fig. 3).

Overall cure rate for patients who had bilateral exploration was $84.4 \%$. The three patients in UNE group who had persistent hypercalcemia, one of them was due to double adenoma, where the other gland was in the contralateral side. The other patient, ultrasound was incorrect in locating the side of the adenoma. The third patient, ultrasound was correctly identified the side of the adenoma, but because it was intrathyroidal, it was missed during the first operation.

\section{Accuracy of Preoperative Ultrasound}

A total of 120 diseased parathyroid glands were evaluated in the study. When ultrasound used to determine the laterality of 


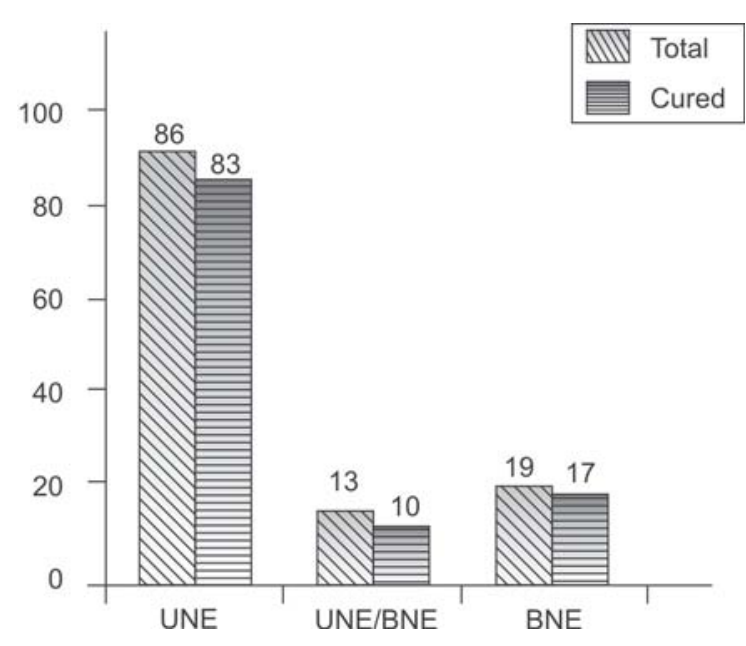

Fig. 3: Cure rate in each surgical procedure group

the enlarged glands, true positive glands were 94 , false positive were 17 , false negative were 26 and true negative were 331 . These give the ultrasound sensitivity of $78.3 \%$, specificity of $95.1 \%$, with the positive predictive value of $84.7 \%$ and negative predictive value of $92.7 \%$. Accuracy of ultrasound was influenced by weight of the glands, $p=0.011$. Evaluation of other factors namely the age of the patients, preoperative calcium and $\mathrm{iPTH}$ and diameter of the glands revealed none was statistically significant (Table 2). When ultrasound correctly identified the side of the disease gland, $81.9 \%$ (77/94) of the time it also correctly discriminate between superior and inferior location of the glands in relation to the thyroid gland.

\section{DISCUSSION}

The customary surgical approach primary HPT was to adopt and explore both sides of the neck coupled with direct visual assessment of all the four parathyroid glands before any decision is made on which gland or glands to be excised. This approach is to prevent or reduce the incidence of persistent or recurrent hypercalcemia in particular in case of double adenoma or multiple glandular disease. Indeed this approach has yielded a high success rate of more than $95 \% .^{1,2}$

Over the years, this approach is beginning to be less favorable mainly because of the fact that majority of primary HPT is due to single hyperfunctioning adenoma. ${ }^{3}$ The argument against it is, if the diseased gland can accurately be localized prior to surgery, the exploration of the predetermined side is possibly sufficient without the need further to explore the opposite side. Furthermore, many surgeons are beginning to prefer unilateral exploration over bilateral approach because of it potential advantages including shorter operating time, avoidance of surgical dissection around the normal parathyroid glands and lesser postoperative morbidities namely recurrent laryngeal nerve injury and postoperative hypocalcemia. ${ }^{4,5}$

Many methods to localize parathyroid adenoma prior to surgery have been used which include ultrasound, sestamibi scintigraphy, computed tomography (CT), magnetic resonance imaging (MRI) and venous sampling. The first two are apparently at present the method of choice. There is sufficient evidence to suggest that combining subtraction sestamibi imaging with high resolution ultrasound will improve sensitivity and give greater information to the surgeon. ${ }^{6-8} \mathrm{CT}$ and MRI are often used in the preoperative evaluation of hyperparathyroid patients, especially when the probability of ectopic glands is high, as in patients with recurrent or persistent hyperparathyroidism. ${ }^{8-10}$ A review by Seehofer et al found that selective venous sampling had higher sensitivity compared to imaging techniques. ${ }^{11}$ but it is rarely used because of its invasive technique. Its main indication is however in redo-operations when two or more preoperative imaging investigations are discordant or unhelpful.

Ultrasound has limitation in accuracy when used alone and is operator dependent. Nevertheless, it has the advantages of being cheap, easy to handle and can be done in clinic setting. Surgeon-performed ultrasound can be done with high accuracy comparable to other ultrasound series published in the literature. Not surprising it may be superior to radiologist performed ultrasound or sestamibi scanning in some institutions. ${ }^{12}$ The

Table 2: Comparison between true positive and false negative diseased glands

\begin{tabular}{llll}
\hline & $\begin{array}{l}\text { True positive } \\
(N=94)\end{array}$ & $\begin{array}{l}\text { False negative } \\
(N=26)\end{array}$ & $50.36 \pm 18.18$ \\
\hline Age (years) mean \pm SD & $49.92 \pm 15.79$ & $3.10(2.64-3.79)$ \\
Preoperative calcium (mmol/L) median (range) & $2.98(2.41-6.50)$ & $52.80(10.00-189.00)$ & $0.395^{* *}$ \\
Preoperative iPTH (pmol/L) median (range) & $37.85(7.60-271.50)$ & $21.0(7.0-40.0)$ & $0.401^{* *}$ \\
Largest diameter (mm) median (range) & $23.0(10.0-50.0)$ & $0.95(0.1-3.8)$ \\
Weight (gm) median (range) & $1.80(0.1-30.6)$ & $0.011^{* *}$ \\
\hline
\end{tabular}

True positive $=$ Enlarged gland seen in ultrasound

False negative $=$ Enlarged gland not seen in ultrasound

*Independent sample T-test.

${ }^{* *}$ Mann-Whitney test. 
parathyroid adenoma is easy to recognize in ultrasound and quite distinct from its neighboring thyroid gland. It is hypoechogenic oval shape with well-circumscribed sharp rim. Thyroid nodule is more echogenic and has more heterogenous echotexture.

We adopted a policy whereby unilateral exploration will be performed only if parathyroid adenoma can be seen in ultrasound. During the exploration, if there were no convincing parathyroid nodule could be found, we would proceed to explore the opposite side of the neck. Our results have shown that when ultrasound was used as the preoperative localizing technique in primary HPT, $72.9 \%$ of the patients had successful unilateral exploration. Out of these, $96.5 \%$ of them were cured. The rate of conversion to bilateral exploration was $13.1 \%$. These clearly demonstrate the obvious benefit of preoperative ultrasound if one decided to practice unilateral exploration for primary HPT patients. The advantage of unilateral exploration is evident as shown in this series where almost $96 \%$ of primary HPT was due to single adenoma. Multiple glandular diseases were seen in 5 patients, 3 double adenomas and 2 four-gland hyperplasia. Two of the double adenoma occurred on the same side of the neck. One of the patients with parathyroid hyperplasia was a lady with known MEN 1 syndrome and she was planned for bilateral exploration irrespective of the ultrasound finding.

Overall cure rate for this study was $93.2 \%$. Surprisingly, the cure rate for patients who underwent bilateral exploration was lower than unilateral approach, $84.4 \%$ and $96.5 \%$ respectively. We postulate that even bilateral exploration might not be able to cure some of primary HPT patients due to the complexity of the disease. The sensitivity and specificity of surgeon performed ultrasound was $78.3 \%$ and $95.1 \%$ respectively. Weight of the glands was found to be statistically significant in influencing accuracy of ultrasound.

In conclusion, we found that unilateral neck exploration with preoperative ultrasound is feasible and the outcome is comparable to bilateral exploration. Seventy-three percent of primary HPT patients may not require bilateral exploration if ultrasound is used prior to surgery with a success rate of more than $96 \%$.

\section{REFERENCES}

1. Udelsman R. Six hundred fifty-six consecutive explorations for primary hyperparathyroidism. Ann Surg 2002;235:665-72.

2. Bilezikian JP, Silverberg SJ. Physiology of the parathyroid glands and pathophysiology of primary hyperparathyroidism. In: Schwartz AE, Pertsemlidis D, Gagner M (Eds) Endocrine Surgery. New York: Marcel Dekker Inc 2004;213-30.

3. Kaplan EL. Thyroid and parathyroid. In: Schwarts SJ (Ed) Principles of Surgery. New York: McGraw-Hill 1989;1613-85.

4. Wang CA. Surgical management of primary hyperparathyroidism. Curr Probl Surg 1985;22:1-50.

5. Tibblin S, Bondeson AG, Ljungberg O. Unilateral parathyroidectomy in hyperparathyroidism due to a single adenoma. Ann Surg 1982;195:245.

6. Grant CS, Thompson G, Farley D, van Heerden J. Primary hyperparathyroidism surgical management since the introduction of minimally invasive parathyroidectomy: Mayo Clinic experience. Arch Surg 2005;140(5):472-79.

7. De Feo ML, Colagrande S, Biagini C, et al. Parathyroid glands: Combination of 99mTc MIBI scintigraphy and US for demonstration of parathyroid glands and nodules. Radiology 2000;214:393-402.

8. Krubsak AJ, Wilson SD, Lawson TL, Collier BD, Hellman RS, Isitman AT. Prospective comparison of radionuclide, computed tomographic, and sonographic localization of parathyroid tumors. World J Surg 1986;10:579-85.

9. Ishibashi M, Nishida H, Hiromatsu Y, Kojima K, Tabuchi E, Hayabuchi N. Comparison of technetium-99m-MIBI, technetium-99m-tetrofosmin, ultrasound and MRI for localization of abnormal parathyroid glands. J Nucl Med 1998;39:320-24.

10. Spritzer CE, Gefter WB, Hamilton R, Greenberg BM, Axel L, Kressel HY. Abnormal parathyroid glands: High-resolution MR imaging. Radiology 1987;162:487-91.

11. Seehofer D, Steinmuller T, Rayes N, et al. Parathyroid hormone venous sampling before reoperative surgery in renal hyperparathyroidism: Comparison with non-invasive localisation procedures and review of the literature. Arch Surg 2004;139:1331-38.

12. Van Husen R, Kim LT. Accuracy of surgeon-performed ultrasound in parathyroid localization. World J Surg 2004;28(11):1122-26. 